OPEN ACCESS

Edited by:

Romesh Khardori,

Eastern Virginia Medical School, USA

Reviewed by:

Andrzej Bartke,

Southern Illinois University

School of Medicine, USA

Ondřej Šeda,

Charles University in Prague,

Czech Republic

${ }^{*}$ Correspondence:

Andrew Charles Adams

a.adams@lilly.com

Specialty section:

This article was submitted to

Diabetes,

a section of the journal

Frontiers in Endocrinology

Received: 18 April 2016

Accepted: 16 June 2016

Published: 30 June 2016

Citation:

Antonellis PJ, Hayes MP and Adams AC (2016) Fibroblast Growth Factor 21-Null Mice Do Not Exhibit an Impaired Response to Fasting.

Front. Endocrinol. 7:77.

doi: 10.3389/fendo.2016.00077

\section{Fibroblast Growth Factor 21-Null Mice Do Not Exhibit an Impaired Response to Fasting}

\author{
Patrick Joseph Antonellis, Meghan Patricia Hayes and Andrew Charles Adams* \\ Lilly Research Laboratories, Lilly Corporate Center, Indianapolis, IN, USA
}

Fibroblast growth factor 21 (FGF21) is a pleotropic metabolic regulator, expression of which is elevated during fasting. To this end, the precise role played by FGF21 in the biology of fasting has been the subject of several recent studies, which have demonstrated contributions to the regulation of both lipid and carbohydrate metabolism. In the present study, we compared wild-type (WT) and FGF21-null (FGF21KO) mice, demonstrating that, despite the significant induction of FGF21 during fasting in the WT animals, our strain of FGF21-null mice exhibits only limited impairments in their adaptation to nutrient deprivation. Specifically, fasted FGF21KO mice display a mild attenuation of gluconeogenic transcriptional induction in the liver accompanied by partially blunted glucose production in response to a pyruvate challenge. Furthermore, FGF21KO mice displayed only minor impairments in lipid metabolism in the fasted state, limited to accumulation of hepatic triglycerides and a reduction in expression of genes associated with fatty acid oxidation. To address the possibility of compensation to germline deletion of FGF21, we further interrogated the role of endogenous FGF21 via acute pharmacological blockade of FGF21 signaling. At the transcriptional level, we show that FGF21 signaling is required for full induction of gluconeogenic and oxidative genes in the liver. However, corroborating our findings in FGF21KO mice, pharmacological blockade of the FGF21 axis did not profoundly disrupt the physiological response to fasting. Taken as a whole, these data demonstrate that, while FGF21 is partially required for appropriate gene expression during the fed to fasted transition, its absence does not significantly impact the downstream physiology of the fasted state.

Keywords: FGF21, KLB, metabolism, fasting, gluconeogenesis

\section{INTRODUCTION}

Fibroblast growth factor 21 (FGF21) is a member of the endocrine subgroup of the FGF family, comprised of FGF19 (FGF15 in mice), FGF21, and FGF23. Unlike the canonical FGFs, members of this family lack a heparin-binding domain, which allows them to enter the circulation (1). Recently, it was demonstrated, through tissue-specific deletion, that the primary source of circulating FGF21 is the liver (2). The systemic physiological effects of FGF21 are mediated by its binding to a receptor complex composed of FGF receptor 1 (FGFR1) and the cofactor protein $\beta$-klotho (KLB) (3). 
While FGFR1 is broadly distributed, KLB is mainly expressed in metabolically active tissues, such as liver, adipose, and pancreas. Thus, it is the expression of KLB, not FGFR1, which restricts the tissues FGF21 can act upon $(4,5)$.

Acute FGF21 administration in mice leads to activation of the FGF signaling pathway in both liver and adipose (6). However, deletion of FGFR1 or KLB, specifically in adipose, demonstrates that adipose tissue activation is intrinsically required for FGF21's pharmacological effects $(7,8)$. In addition to these peripheral sites of action, it has recently been proposed that FGF21 may act centrally (9). Concordant with this assertion, expression of KLB has been reported in discrete areas of the hypothalamus, deletion of which has been reported to abrogate the metabolic effects of exogenous FGF21 administration (10). It has also been demonstrated that central FGF21 activates the sympathetic nervous system and that intact sympathetic tone is required to mediate its effects at the periphery $(11,12)$.

In the liver, FGF21 expression primarily resides downstream of the transcription factor, peroxisome proliferator-activated receptor alpha (PPAR $\alpha)$ (13). PPAR $\alpha$ and, subsequently, FGF21 are induced in states that demand enhanced hepatic lipid oxidation, such as fasting and ketogenic diet feeding $(14,15)$. Interestingly, FGF21-null (FGF21KO) mice have previously been reported to exhibit impairments in glucose and lipid metabolism (16). Furthermore, in response to feeding of a ketogenic diet, both global deletion and liver-specific FGF21 knockdown lead to hepatic triglyceride accumulation, accompanied by an attenuation of hepatic oxidative gene expression $(14,17)$. In addition to defects in lipid metabolism, FGF21 KO mice also exhibit enhanced adipose mass coupled with the development of larger adipocytes (17-19). Conversely, FGF21 transgenic mice (FGF21Tg) are leaner than their WT counterparts, with a concomitant increase in hepatic fatty acid oxidation $(15,16)$. In agreement with the phenotype of FGF21Tg animals, pharmacologic treatment with FGF21 leads to a significant decrease in body mass in preclinical models $(20,21)$. This reduction in body mass is primarily due to decreased adipose mass, thought to be driven in large part by significantly increased energy expenditure and a reduction in respiratory exchange ratio (RER), which when taken together are suggestive of a state of elevated lipid oxidation (20).

In the present study, we utilize both genetic and pharmacological approaches to assess the physiological role of FGF21 during fasting. We demonstrate that, while our own strain of FGF21KO mice do exhibit impairments in the transcriptional regulation of pathways involved in lipid and carbohydrate metabolism during fasting, these defects had little in the way of physiological consequences. To further interrogate the physiological role of FGF21 during fasting and to address the potential for confounding developmental compensation in the FGF21KO mice, we sought to directly inhibit endogenous FGF21 signaling. To do so, we utilized a variant of the wildtype human FGF21 protein lacking $17 \mathrm{~N}$-terminal amino acid residues, termed $\Delta \mathrm{N} 17$, which we have previously shown blocks FGF21 action in vivo (22). In agreement with the effects observed in FGF21KO mice, we found that treatment with $\Delta \mathrm{N} 17$ either during the early fed to fasted transition or after a prolonged fast did significantly impact the transcriptional response, yet led to only minor physiological effects. Taken as a whole, these studies demonstrate that, while manipulation of the FGF21 axis can modify the transcriptional response to caloric deprivation, FGF21 is not explicitly required for an appropriate, integrated, physiological response to fasting.

\section{MATERIALS AND METHODS}

\section{Proteins}

Human FGF21 $\Delta$ N17 was generated as previously described (23).

\section{Animals}

All animals were individually housed in a temperature-controlled $\left(24^{\circ} \mathrm{C}\right)$ facility with $12 \mathrm{~h} / 12 \mathrm{~h}$ light/dark cycle. Animal protocols in this study were approved by the Eli Lilly and Co., Animal Use and Care Committee (Protocol No. 13-030).

\section{Comparison of WT and FGF21KO Animals in the Fed and Fasted States}

Male C57Bl/6J and FGF21 knockout (FGF21KO) mice (Taconic Farms) were maintained on a standard chow diet consisting of $13 \%$ fat, $67 \%$ carbohydrate, and $20 \%$ protein caloric content (2014; Harlan Teklad, Madison, WI, USA) and had free access to food and water before randomization by weight. A cohort of mice from each genotype had their food removed at the beginning of the light cycle and were sacrificed following a $24 \mathrm{~h}$ fast. A separate cohort from each genotype was allowed ad libitum access to food and sacrificed at the end of the dark cycle. Prior to sacrifice, blood glucose levels were assessed using handheld glucometers (Accu-Chek Aviva; Roche Diagnostics, Indianapolis, IN, USA). Following sacrifice, liver and epididymal white adipose tissue (WAT) samples were rapidly dissected and flash frozen in liquid nitrogen. Whole blood was collected in EDTA coated tubes, centrifuged, and plasma collected for analysis. Each cohort consisted of at least six mice.

\section{Acute Treatment of WT Animals}

Male C57Bl/6J mice (Taconic Farms) 11-12 weeks of age were maintained on a standard chow diet (2014; Harlan Teklad). At the beginning of the light cycle, mice had their food removed and dosed with either $5 \mathrm{mg} / \mathrm{kg}$ of $\Delta \mathrm{N} 17$ or vehicle $v i a$ intraperitoneal (IP) injection. Number of injections and duration of study were as indicated. At the indicated time points, blood glucose was measured using handheld glucometers (Accu-Chek Aviva; Roche Diagnostics). At the end of each study, mice were sacrificed, and liver and WAT samples were rapidly dissected and flash frozen in liquid nitrogen. Whole blood was collected in EDTA-coated tubes, centrifuged, and plasma removed for analysis. Six mice were used for each treatment group.

\section{Pyruvate Tolerance Test}

Mice were fasted for the indicated amount of time. Following the fast, animals were administered $2 \mathrm{~g} / \mathrm{kg}$ sodium pyruvate via IP injection. Blood glucose was measured at the indicated time points using handheld glucometers (Accu-Chek Aviva; Roche Diagnostics). 


\section{Metabolites and Hormones}

Plasma triglycerides and free fatty acids were measured using a Hitachi 912 Clinical Chemistry analyzer (Roche Diagnostics). To measure liver triglyceride content, a 10\% tissue homogenate was prepared and analyzed using a Hitachi 912 Clinical Chemistry analyzer. Circulating levels of insulin (Ultra Sensitive Mouse Insulin ELISA; CrystalChem, Downers Grove, IL, USA) and FGF21 (Mouse/Rat FGF21 Quantikine ELISA; R\&D Systems, Minneapolis, MN, USA) were determined by ELISA assay. Colorimetric assays were used to determine plasma $\beta \mathrm{HB}$ ( $\beta$-hydroxybutyrate LiquiColor; Stanbio Laboratories, Boerne, TX, USA) and liver glycogen content (Glycogen Assay Kit; Sigma-Aldrich, St. Louis, MO, USA).

\section{Protein Measurements}

Protein was extracted from tissue using mammalian protein extraction reagent (M-PER) (Pierce, Rockford, IL, USA) containing $1 \times$ halt protease and phosphatase inhibitor cocktail (Pierce). FGF21 protein concentration was determined by ELISA (Mouse/ Rat FGF21 Quantikine ELISA; R\&D Systems). The average minimum detectable dose of the assay is $3.81 \mathrm{pg} / \mathrm{mL}$, and all samples measured fell within the linear range of 31.3-2000 pg/ $\mathrm{mL}$. Total and phosphorylated hormone-sensitive lipase (HSL) was determined by Western blot. Briefly, WAT protein extracts were separated by SDS-PAGE and transferred to nitrocellulose membranes, which were probed using anti-rabbit antibodies to total and phosphorylated HSL (Cell Signaling Technologies, Danvers, MA, USA).

\section{RNA Isolation, RT, and Real-time Quantitative PCR}

RNA was isolated from tissues using TRIzol reagent (Invitrogen, Carlsbad, CA, USA) and the RNeasy lipid mini kit (Qiagen, Venlo, Netherlands) and then reverse transcribed into cDNA using the Quantitect Reverse Transcription Kit (Qiagen). Reactions were performed on an Applied Biosystems QuantStudio 7 RealTime PCR System (Applied Biosystems, South San Francisco, CA, USA), CT values were normalized to $\beta$-actin, and relative expression was calculated by the $\Delta \Delta \mathrm{CT}$ method. Fold change was calculated by normalizing relative expression to the proper control, WT-fed or PBS-treated animals. Assays-on-Demand Gene expression products (Applied Biosystems) were as follows: CPT1a Mm01231183-m1; ACADS Mm00431617_m1; ACADM Mm01323360_g1; ACADL Mm00599660_m1; ACADVL Mm00 44293_m1; HADH Mm00492535_m1; CD36 Mm01135198_m1; G6PC Mm00839363-m1; PCK1 Mm01247058-m1; HMGCS2 Mm00550050_m1; BDH1 Mm00558330_m1; PGC1 $\alpha$ Mm01 208835-m1; CIDEA Mm00432554_m1; UCP1 Mm01244861_m1; FASN Mm00662319_m1; HSL Mm00495359_m1; ATGL Mm00 503040_m1; LPL Mm00434770_m1; and FGF21 Mm00840165_g1.

\section{Statistical Analysis}

Data are presented as mean \pm SEM. Statistical analysis was performed using two-way ANOVA or one-way ANOVA followed by Dunnett's multiple comparisons test or $T$-test where appropriate. Differences were considered significant when $P<0.05$.

\section{RESULTS}

\section{FGF21KO Mice Exhibit Modest Alterations in Lipid and Carbohydrate Metabolism during a Fast}

To assess the role of FGF21 during the adaptive response to fasting, we compared cohorts of WT and FGF21KO mice in both the fed state and following a 24-h fast. We found that increased plasma levels of FGF21 during fasting (Figure 1A) correlated with a significant elevation in FGF21 mRNA (Figure 1B) and protein (Figure 1C) in the liver, but not adipose tissue (Figures 1B,C), consistent with recent findings (2). Both WT and FGF21KO animals exhibited expected reductions in blood glucose (Figure 1D) and insulin (Figure 1E). However, while FGF21KO animals did appear to have mildly reduced blood glucose levels compared to WT mice in the fasted state, this effect was not statistically significant (Figure 1D).

It has been demonstrated that FGF21 is important for the induction of ketosis when animals are maintained on a ketogenic diet $(14,16)$. However, consistent with previous studies (17), our strain of FGF21KO animals fed on a chow diet did not show impaired $\beta \mathrm{HB}$ production in the fasted state (Figure 1F). Nor were circulating triglycerides altered in either genotype (Figure 1G). Interestingly, plasma free fatty acids were elevated in fasted WT, but not FGF21KO animals (Figure 1H). Therefore, as FGF21 has been implicated in the regulation of lipolysis (18), we investigated whether altered lipase activity in the WAT of the FGF21 KO animals was responsible for this discrepancy. We found no differences in the expression of HSL or lipoprotein lipase (LPL); however, while induction of adipose triglyceride lipase (ATGL) in the fasted state did not reach significance in FGF21KO mice, it did trend upward to a similar degree as observed in the WT animals (Figure 2B). Furthermore, during fasting, we observed equivalent phosphorylation of HSL in adipose tissue of WT and FGF21KO animals (Figure 2C). In addition to mediators of lipolysis, we assessed the known FGF21 target genes peroxisome proliferator-activated receptor gamma coactivator 1 -alpha $(\mathrm{PGC} 1 \alpha)$ and uncoupling protein 1 (UCP1) and found no differences in their expression between the genotypes (Figure 2B).

While both WT and FGF21KO mice exhibited elevated hepatic triglyceride content following a fast, FGF21KO animals accumulated significantly more than their WT counterparts (Figure 1I). As we found no evidence of enhanced lipolysis in adipose tissue, we next examined the expression of genes associated with fatty acid import and oxidation in the liver. Importantly, we found that the induction of genes involved in fatty acid oxidation (carnitine palmitoyltransferase 1A, CPT1a; acyl-Coenzyme A dehydrogenase, short chain, ACADs; acyl-Coenzyme A dehydrogenase, medium chain, ACADM; acyl-Coenzyme A dehydrogenase, long chain, ACADL; acyl-Coenzyme A dehydrogenase, very long chain, ACADVL) were attenuated in FGF21KO animals (Figure 2A). Additionally, FGF21KO animals exhibited a significantly greater increase in expression of the fatty acid transporter, CD36, in the fasted state (Figure 2A).

Next, we sought to determine the impact of global FGF21 deletion on carbohydrate metabolism during fasting. We found that, while there was no difference in glucose-6-phosphatase (G6PC) 

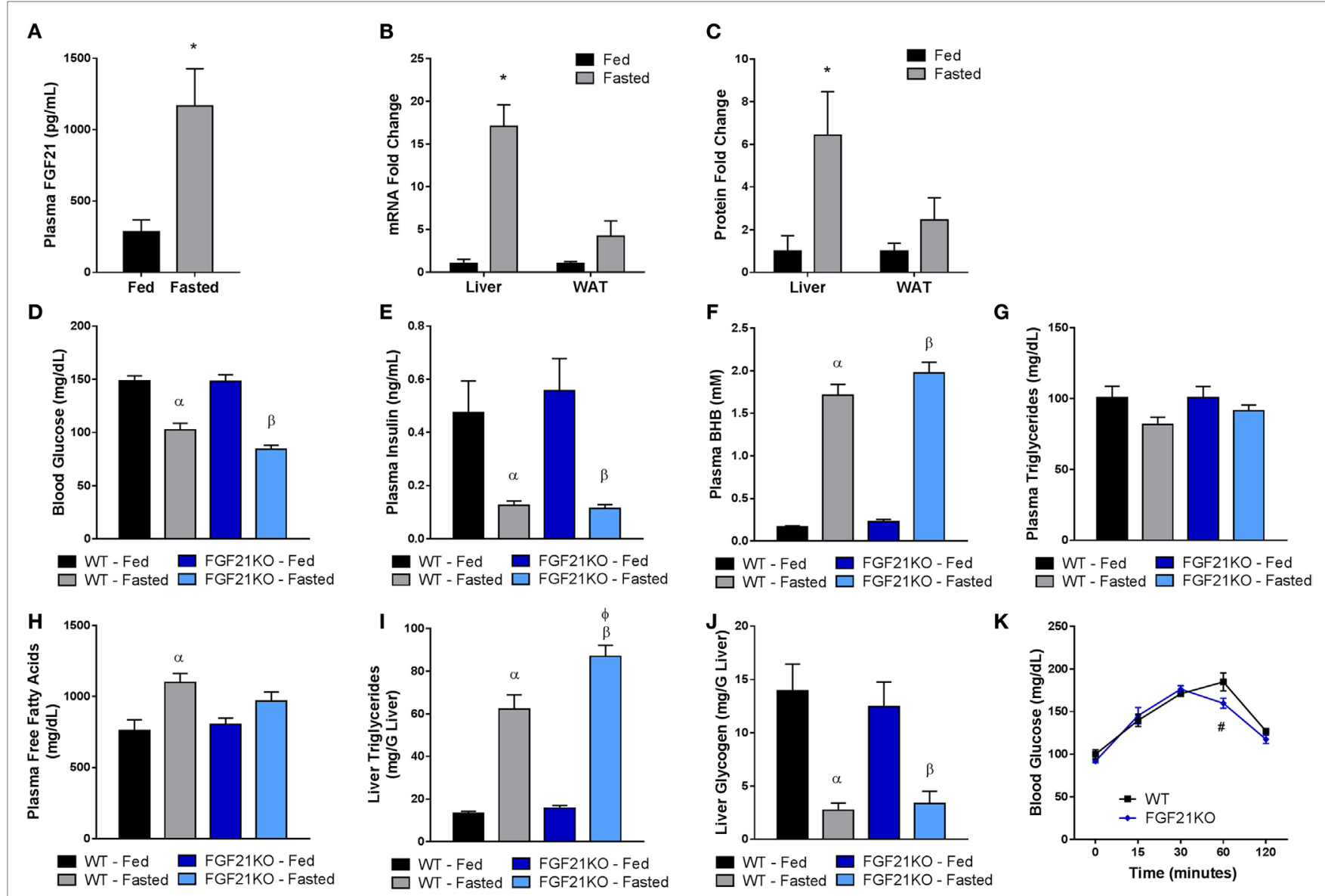

FIGURE 1 | FGF21 is primarily expressed in the liver and is dispensable for the physiological response to fasting. WT and FGF21KO animals were either allowed ad libidum access to food (fed) or subjected to a 24-h fast (fasted). (A) Plasma FGF21 levels were measured in WT animals. (B) FGF21 mRNA and (C) protein expression were measured in liver and adipose tissue from WT animals. (D) Blood glucose was assessed via glucometer in each cohort. Plasma was collected from WT and FGF21KO animals and used to determine circulating levels of (E) insulin, (F) $\beta H B$, (G) triglycerides, and (H) free fatty acids. The concentration of (I) triglycerides and $(\mathbf{J})$ glycogen was measured in the livers of WT and FGF21KO animals. (K) A pyruvate tolerance test was performed on a separate cohort of WT and FGF21KO animals by administration of $2 \mathrm{~g} / \mathrm{kg}$ sodium pyruvate following a $22-\mathrm{h}$ fast. ${ }^{*} p<0.05$ vs. fed; $\alpha, p<0.05$ vs. WT Fed; $\beta, p<0.05$ vs. FGF21KO fed; $\varphi, p<0.05$ vs. WT fasted; \#, $p<0.05$ vs. WT. No statistical difference was observed in fed measurements between genotypes.

expression, induction of phosphoenolpyruvate carboxykinase 1 (PCK1) during fasting was significantly attenuated in FGF21KO mice (Figure 2A). To assess whether the observed attenuation in PCK1 gene expression in FGF21KO mice impacted gluconeogenic capacity, we conducted a pyruvate tolerance test (PTT). To our surprise, we found that FGF21KO mice exhibited only a modest reduction in gluconeogenesis following a pyruvate challenge (Figure 1K). This minor effect on carbohydrate metabolism was reflected in maintenance of normal hepatic glycogen content in the FGF21KO animals (Figure 1J).

\section{Pharmacological Inhibition of Endogenous FGF21 Signaling Has No Effect on Lipid and Carbohydrate Utilization during the Early Fed to Fasted Transition}

To investigate the potential role of endogenous FGF21 during the transition from the fed to fasted state and to mitigate potential concerns regarding developmental compensation to germline deletion of FGF21, we subjected WT animals to a time course of FGF $21-\Delta$ N17 $(\Delta$ N17) treatment. Since $\Delta$ N17 functions as an antagonist of FGF21 action in vivo (22), pharmacological administration of the protein allows for acute blockade of the FGF21 axis. At the beginning of the light cycle, animals had their food removed, then were dosed with $\Delta \mathrm{N} 17$ every $3 \mathrm{~h}$ for $12 \mathrm{~h}$. During this initial transition period, treatment with $\Delta \mathrm{N} 17$ had no significant effect on blood glucose (Figure 3A) or plasma insulin levels (Figure 3B). Additionally, there was no effect on circulating levels of $\beta \mathrm{HB}$ (Figure 3C), triglycerides (Figure 3D), free fatty acids (Figure 3E), hepatic triglyceride (Figure 3F), or liver glycogen content (Figure 3G). When gene expression was assessed in the liver, we found that of the genes involved in fatty acid oxidation (CPT1a, ACADS, ACADM, ACADVL, HADH), only HADH was significantly altered by $\Delta \mathrm{N} 17$ treatment (Figure $3 \mathbf{H}$ ). Similarly, genes related to gluconeogenesis (G6PC, PCK1) and ketone body production (3-hydroxybutyrate dehydrogenase, type $1, \mathrm{BDH} 1$; 
A

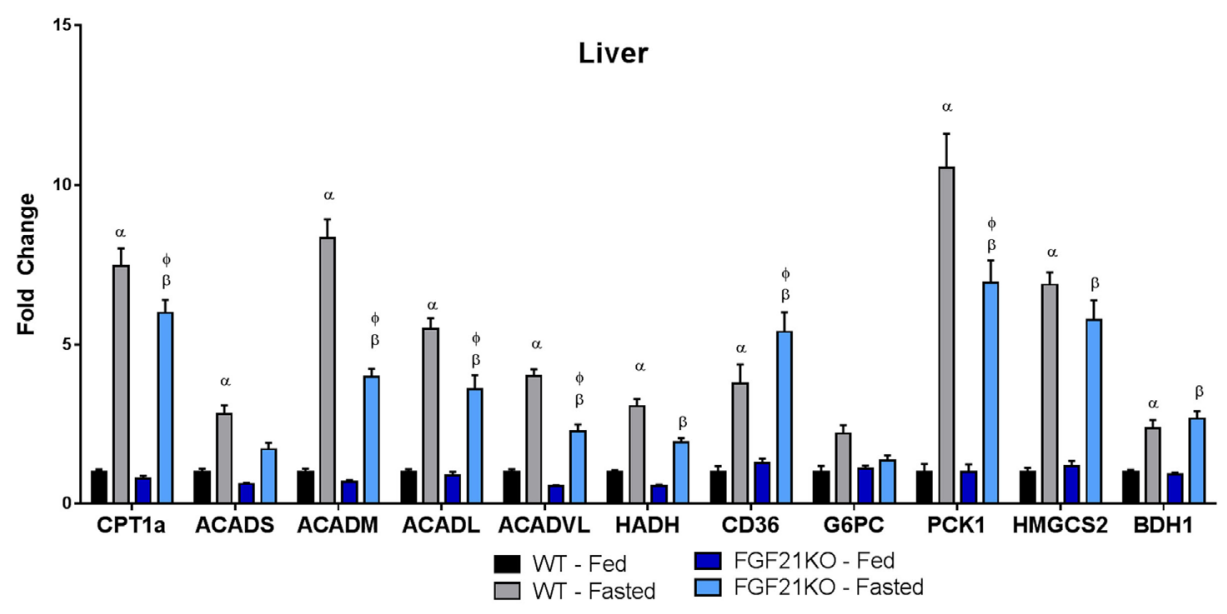

B

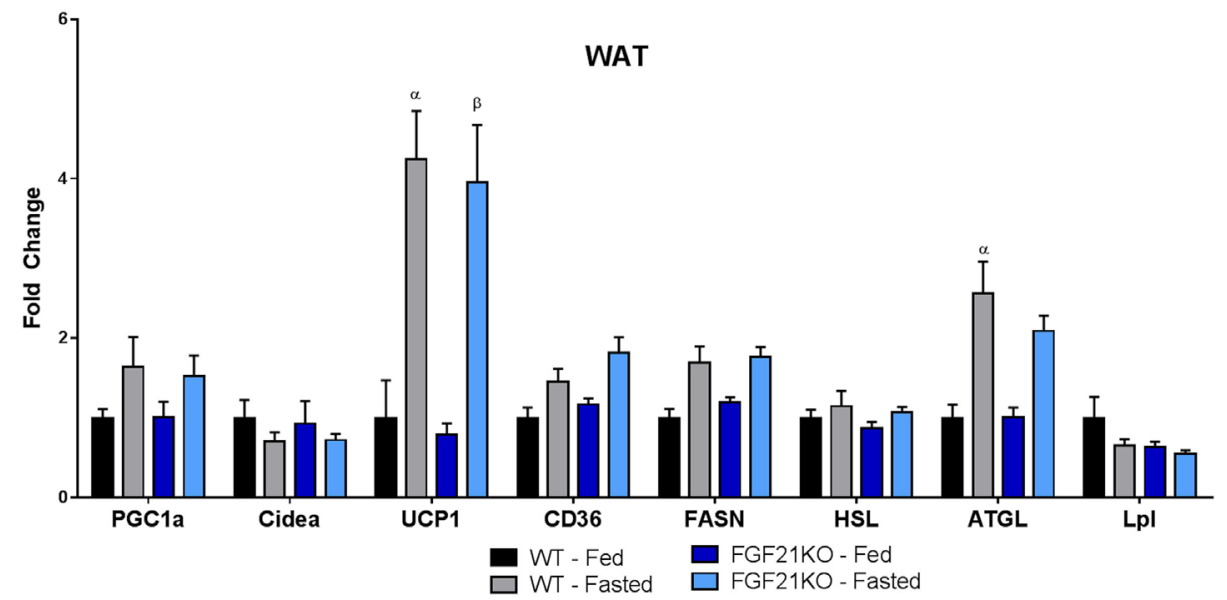

C

Fed

Fasted

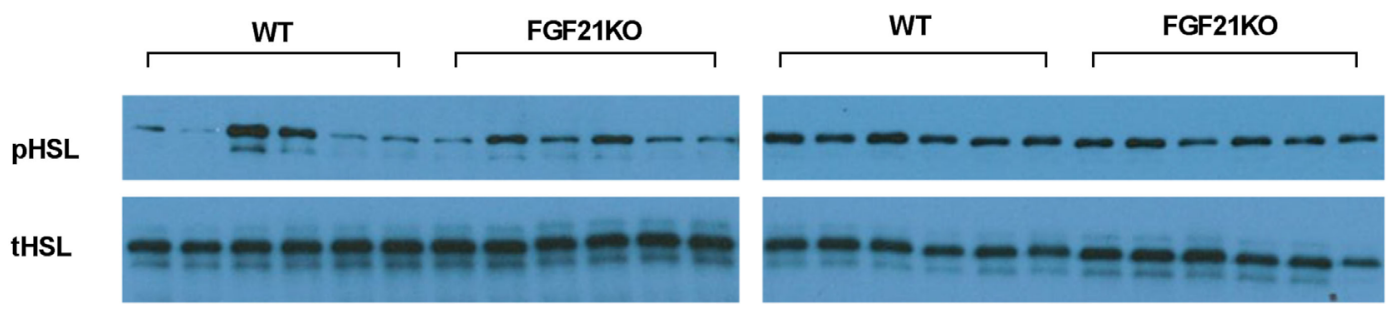

FIGURE 2 | Oxidative and gluconeogenic gene expression is attenuated in the liver, while lipase gene expression and activity in WAT remains unaltered in fasted FGF21KO mice. (A) In the liver, expressions of genes associated with fatty acid oxidation (CPT1a, ACADS, ACADM, ACADL, ACADVL, $\mathrm{HADH}$ ), fatty acid import (CD36), gluconeogenesis (G6PC, PCK1), and ketone body production (HMGCS2, BDH1) were measured. (B) Expressions of genes associated with lipolysis (HSL, ATGL, LPL), fatty acid synthesis (FASN), fatty acid import (CD36), and thermogenesis (CIDEA, UCP1) were measured. In adipose tissue: $\alpha, p<0.05$ vs. WT Fed; $\beta, p<0.05$ vs. FGF21KO fed; $\varphi, p<0.05$ vs. WT fasted. No statistical differences were observed between genotypes in fed expression of any gene measured. (C) The amount of total and phosphorylated HSL was assessed by Western blot in WAT of WT and FGF21KO animals in both the fed and fasted state.

3-hydroxy-3-methylglutaryl-CoA synthase 2, HMGCS2) were unaffected (Figure $\mathbf{3 H}$ ). Somewhat surprisingly, in the WAT of animals treated with $\Delta \mathrm{N} 17$, we did observe reduced expression of PGC1 $\alpha$ (Figure 3I). These data lead us to conclude that endogenous FGF21 has a limited role during early fasting, supportive of earlier studies, which demonstrated that full induction of hepatic and serum FGF21 requires prolonged fasting $(14,15)$.
Interruption of FGF21 Signaling during Prolonged Fasting Does Not Significantly Impact Lipid or Carbohydrate Metabolism

To assess the role of FGF21 in the regulation of gluconeogenesis during late fasting, WT animals were fasted for $21 \mathrm{~h}$, given a single injection of $\Delta \mathrm{N} 17$, and then subjected to a PTT. Consistent 


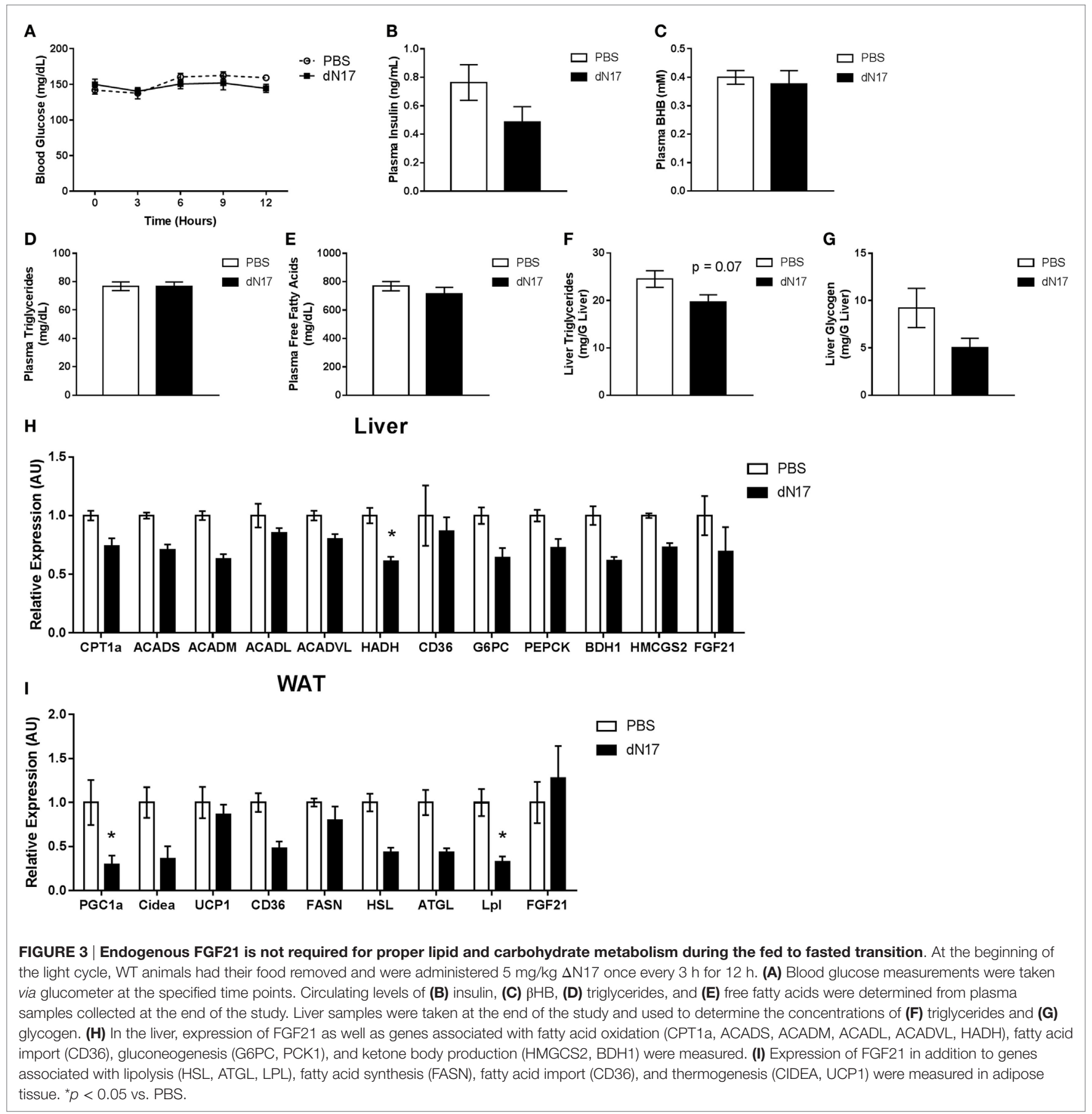

with our earlier studies in the FGF21KO mice, we found that $\Delta \mathrm{N} 17$ treatment had no effect on gluconeogenesis following a pyruvate challenge (Figure 4A). To determine whether other discrete aspects of the fasted response were impacted by inhibition of FGF21 signaling, a separate cohort of 23-h fasted mice was treated with a single injection of $\Delta \mathrm{N} 17$ and sacrificed $1 \mathrm{~h}$ later. In agreement with our previous findings, acute treatment with $\Delta \mathrm{N} 17$ had no significant effect on circulating glucose (Figure 4B), insulin (Figure 4C), $\beta \mathrm{HB}$ (Figure 4D), or free fatty acid (Figure 4F) levels. Mice treated with $\Delta$ N17 had significantly elevated plasma triglycerides (Figure 4E); however, both hepatic triglyceride (Figure 4G) and glycogen (Figure $\mathbf{4 H}$ ) content were unchanged by $\Delta \mathrm{N} 17$ treatment. The gene expression profile in the $\Delta \mathrm{N} 17$-treated animals exhibited trends reflective of the results observed in the FGF21KO mice; however, none of these differences reached statistical significance in the liver (Figure 4I) or adipose (Figure 4J), suggesting that chronic changes to FGF21 tone may be required to observe the full magnitude of its effects. 


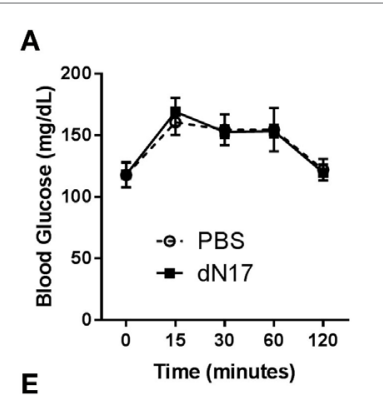

E

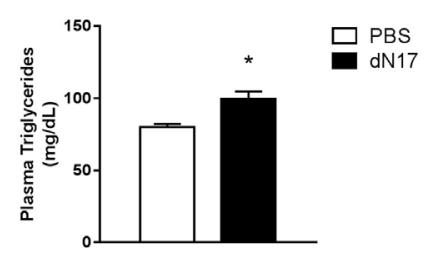

I

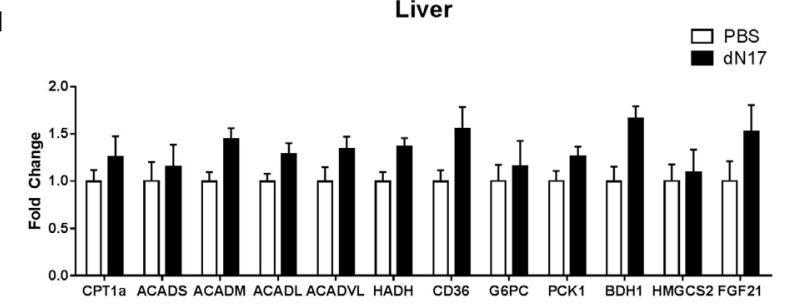

\section{B}

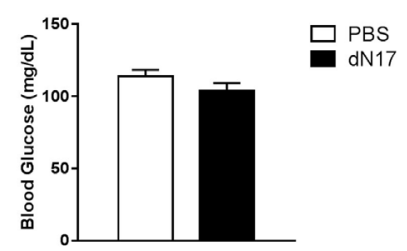

F

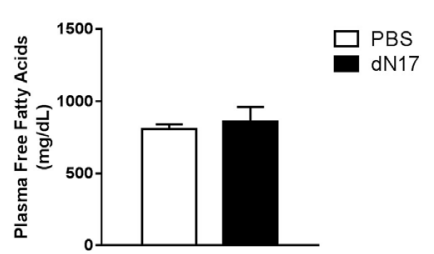

C

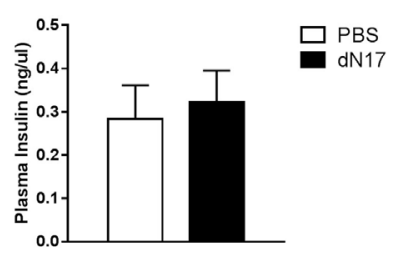

G

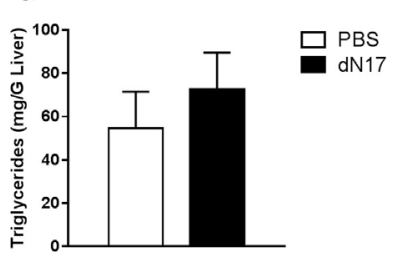

D

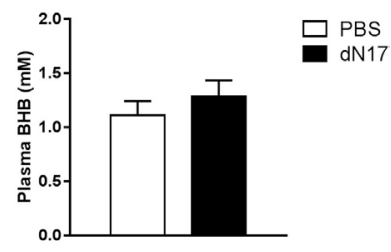

H

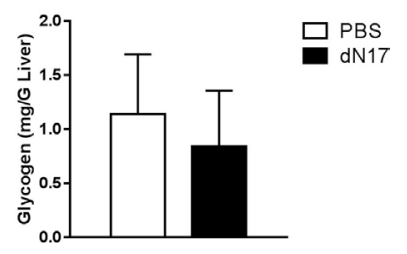

WAT

$\mathbf{J}$
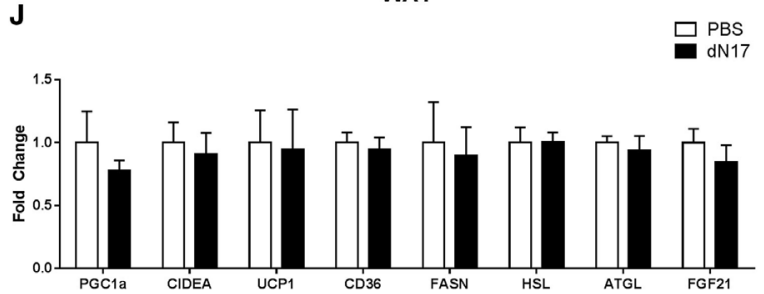

FIGURE 4 | Blockade of FGF21 signaling during late fasting does not impair the fasted response. WT mice were fasted for $21 \mathrm{~h}$, administered $5 \mathrm{mg} / \mathrm{kg}$ $\Delta \mathrm{N} 17$, and then subjected to a PTT $1 \mathrm{~h}$ later by administration of $2 \mathrm{~g} / \mathrm{kg}$ sodium pyruvate. (A) Blood glucose was measured at the specified time points via glucometer. A separate cohort of WT mice was fasted for $23 \mathrm{~h}$, administered $5 \mathrm{mg} / \mathrm{kg} \Delta \mathrm{N} 17$, and then sacrificed $1 \mathrm{~h}$ later. (B) Blood glucose was assessed via glucometer. Plasma samples were collected and used to determine circulating levels of (C) insulin, (D) $\beta$ HB, (E) triglycerides, and (F) free fatty acids. Hepatic (G) Triglyceride and (H) glycogen content was measured. (I) In the liver, expression of FGF21 as well as genes associated with fatty acid oxidation (CPT1a, ACADS, ACADM, ACADL, ACADVL, HADH), fatty acid import (CD36), gluconeogenesis (G6PC, PCK1), and ketone body production (HMGCS2, BDH1) were measured. (J) Expression of FGF21 in addition to genes associated with lipolysis (HSL, ATGL, LPL), fatty acid synthesis (FASN), fatty acid import (CD36), and thermogenesis (CIDEA, UCP1) were measured in adipose tissue. ${ }^{*} p<0.05$ vs. PBS.

\section{DISCUSSION}

Despite significant study, including the generation of three separate FGF21-null models (16-18), the physiological role of FGF21 remains uncertain. Broadly, it has been proposed that a primary role of FGF21 is to coordinate lipid and carbohydrate metabolism during nutrient deprivation. In support of this view, FGF21 has been shown to be induced by nutrientdeficient states such as prolonged fasting as well as during exposure to ketogenic (17) and protein-deficient diets (24-26). In the present study, we investigated the role of FGF21 in the response to fasting through examination of our own strain of FGF21KO animals, in addition to pharmacological inhibition of endogenous FGF21 action.

Congruent with previously published findings (14), we show that, following a 24-h fast, plasma concentrations of FGF21 were indeed significantly elevated in WT mice. This increase in circulating FGF21 corresponded with a significant elevation in FGF21 mRNA and protein in the liver, but not adipose, supportive of a recent report describing the liver as the primary source of endocrine FGF21 (2). We went on to extend these findings by demonstrating that, while FGF21KO mice were able to maintain glycemia in a manner equivalent to their WT counterparts, they did exhibit defects in lipid metabolism during fasting. The elevation of circulating free fatty acids in the fasted state observed in WT mice was abolished in FGF21KO animals despite both genotypes having similar HSL abundance and activity in WAT. Interestingly, in addition to reduced serum FFA levels, the FGF21-null animals also exhibited elevated hepatic triglyceride accumulation in the fasted state. When hepatic gene expression was assessed, we found attenuation in the induction of genes involved in fatty acid oxidation as well as increased expression of the fatty acid transporter, CD36. Therefore, we suggest that reduced oxidative capacity coupled with increased fatty acid import in the liver may serve to explain both the attenuation of free fatty acids in circulation and the increased hepatic triglyceride content observed in FGF21KO mice during fasting conditions. In support of this conclusion, it has been demonstrated that hepatic CD36 correlates with dyslipidemia in diet-induced obese animals; furthermore, overexpression of CD36 in the liver leads to increased fatty acid uptake and triglyceride storage in the liver (27). It has also been shown that reducing fatty acid oxidation through the use of a specific inhibitor of liver CPT1 causes hepatic triglyceride accumulation (28). Thus, the coupling of these two 
mechanisms would serve as a mechanistic basis for the impaired lipid homeostasis observed in our strain of FGF21KO mice.

The primary manner in which FGF21 is thought to regulate glucose metabolism is via modulation of hepatic gluconeogenesis (29). In support of this contention, FGF21-null animals have been reported to exhibit attenuated hepatic gluconeogenic gene expression in the fasted state (16). Furthermore, it has also been shown that this transcriptional defect translates to a functional impairment in gluconeogenesis following a fast (30). However, here, we show that despite attenuation in gluconeogenic gene expression in the liver, our own strain of FGF21KO animals had no significant impairment of gluconeogenesis, exhibiting nearly identical endogenous glucose production as WT mice. These results are in agreement with the phenotype reported in a third strain of FGF21KO mice (31).

Given the discordance between the three strains of FGF21KO mice and to avoid potential confounding effects of compensation due to germline deletion, we further interrogated the role of the FGF21 axis in the fasted state, pharmacologically. Specifically, we inhibited endogenous FGF21 through the use of a truncated form of the protein $(\Delta \mathrm{N} 17)$ during both the early and late phases of the fed to fasted transition. Treatment with $\Delta \mathrm{N} 17$ had little effect on early fasting, which given the short nature of the fast $(12 \mathrm{~h})$ and that animals would not normally be eating much during this time, is not wholly unexpected. However, in 24-h-fasted animals, we found that interruption of FGF21 signaling led to little more than a blunting of the transcriptional response to fasting. Indeed, in spite of a diminished transcriptional response, $\Delta$ N17 treatment had no effect on hepatic gluconeogenesis in response to a pyruvate challenge nor did it significantly alter systemic lipid

\section{REFERENCES}

1. Kharitonenkov A. FGFs and metabolism. Curr Opin Pharmacol (2009) 9:805-10. doi:10.1016/j.coph.2009.07.001

2. Markan KR, Naber MC, Ameka MK, Anderegg MD, Mangelsdorf DJ, Kliewer SA, et al. Circulating FGF21 is liver derived and enhances glucose uptake during refeeding and overfeeding. Diabetes (2014) 63:4057-63. doi:10.2337/ db14-0595

3. Ogawa Y, Kurosu H, Yamamoto M, Nandi A, Rosenblatt KP, Goetz R, et al. BetaKlotho is required for metabolic activity of fibroblast growth factor 21. Proc Natl Acad Sci U S A (2007) 104:7432-7. doi:10.1073/pnas.0701600104

4. Kharitonenkov A, Dunbar JD, Bina HA, Bright S, Moyers JS, Zhang C, et al. FGF-21/FGF-21 receptor interaction and activation is determined by betaKlotho. J Cell Physiol (2008) 215:1-7. doi:10.1002/jcp.21357

5. Adams AC, Cheng CC, Coskun T, Kharitonenkov A. FGF21 requires betaklotho to act in vivo. PLoS One (2012) 7:e49977. doi:10.1371/journal. pone.0049977

6. Fisher FM, Estall JL, Adams AC, Antonellis PJ, Bina HA, Flier JS, et al. Integrated regulation of hepatic metabolism by fibroblast growth factor 21 (FGF21) in vivo. Endocrinology (2011) 152:2996-3004. doi:10.1210/ en.2011-0281

7. Ding X, Boney-Montoya J, Owen BM, Bookout AL, Coate KC, Mangelsdorf DJ, et al. betaKlotho is required for fibroblast growth factor 21 effects on growth and metabolism. Cell Metab (2012) 16:387-93. doi:10.1016/j.cmet.2012.08.002

8. Adams AC, Yang C, Coskun T, Cheng CC, Gimeno RE, Luo Y, et al. The breadth of FGF21's metabolic actions are governed by FGFR1 in adipose tissue. Mol Metabol (2013) 2:31-7. doi:10.1016/j.molmet.2012.08.007

9. Sarruf DA, Thaler JP, Morton GJ, German J, Fischer JD, Ogimoto K, et al. Fibroblast growth factor 21 action in the brain increases energy expenditure homeostasis. These results were reminiscent of the physiological effects observed in two of the three strains of FGF21KO mice; however, they did not fully recapitulate the phenotype observed in those animals. It is possible that transcriptional changes during later stages of fasting are required for FGF21 to exert its physiological effects; therefore, longer duration of $\Delta \mathrm{N} 17$ coverage would be required. Alternatively, the nature of endogenous FGF21 action may exist not under the conditions of prolonged fasting, but instead become manifest in a state of true starvation. Thus, longer periods of nutrient deprivation ( $>24 \mathrm{~h}$ ) followed by $\Delta$ N17 administration may illuminate the biological processes under the control of elevated FGF21 during fasting.

While it appears that FGF21 is required for a full transcriptional response to fasting, the physiological consequences of its loss are extremely limited in nature. Furthermore, those physiological differences we did observe were related specifically to the uptake and subsequent oxidation of hepatic lipids. Taken as a whole, these findings support the view that FGF21 does not play a meaningful role in the mobilization of energy stores during nutrient deprivation, but, instead, acts in an autocrine manner in the fasted state to facilitate hepatic lipid homeostasis. We suggest that, given that the transcriptional changes are consistent in all three lines of FGF21KO mice, the range of physiological responses observed may be due to the environment in which the mice are housed or the variance in the specific experimental conditions of the studies.

\section{AUTHOR CONTRIBUTIONS}

PA, MH, and AA designed and executed the studies and also analyzed the data and wrote the manuscript.

and insulin sensitivity in obese rats. Diabetes (2010) 59:1817-24. doi:10.2337/ db09-1878

10. Bookout AL, De Groot MH, Owen BM, Lee S, Gautron L, Lawrence HL, et al. FGF21 regulates metabolism and circadian behavior by acting on the nervous system. Nat Med (2013) 19:1147-52. doi:10.1038/nm.3249

11. Owen BM, Ding X, Morgan DA, Coate KC, Bookout AL, Rahmouni K, et al. FGF21 acts centrally to induce sympathetic nerve activity, energy expenditure, and weight loss. Cell Metab (2014) 20:670-7. doi:10.1016/j. cmet.2014.07.012

12. Douris N, Stevanovic DM, Fisher FM, Cisu TI, Chee MJ, Nguyen NL, et al. Central fibroblast growth factor 21 browns white fat via sympathetic action in male mice. Endocrinology (2015) 156:2470-81. doi:10.1210/en.2014-2001

13. Adams AC, Kharitonenkov A. FGF21: the center of a transcriptional nexus in metabolic regulation. Curr Diabetes Rev (2012) 8:285-93. doi:10.2174/157339912800840505

14. Badman MK, Pissios P, Kennedy AR, Koukos G, Flier JS, Maratos-Flier E. Hepatic fibroblast growth factor 21 is regulated by PPARalpha and is a key mediator of hepatic lipid metabolism in ketotic states. Cell Metab (2007) 5:426-37. doi:10.1016/j.cmet.2007.05.002

15. Inagaki T, Dutchak P, Zhao G, Ding X, Gautron L, Parameswara V, et al. Endocrine regulation of the fasting response by PPARalpha-mediated induction of fibroblast growth factor 21. Cell Metab (2007) 5:415-25. doi:10.1016/j. cmet.2007.05.003

16. Potthoff MJ, Inagaki T, Satapati S, Ding X, He T, Goetz R, et al. FGF21 induces PGC-1alpha and regulates carbohydrate and fatty acid metabolism during the adaptive starvation response. Proc Natl Acad Sci U S A (2009) 106:10853-8. doi:10.1073/pnas.0904187106

17. Badman MK, Koester A, Flier JS, Kharitonenkov A, Maratos-Flier E. Fibroblast growth factor 21-deficient mice demonstrate impaired 
adaptation to ketosis. Endocrinology (2009) 150:4931-40. doi:10.1210/ en.2009-0532

18. Hotta Y, Nakamura H, Konishi M, Murata Y, Takagi H, Matsumura S, et al. Fibroblast growth factor 21 regulates lipolysis in white adipose tissue but is not required for ketogenesis and triglyceride clearance in liver. Endocrinology (2009) 150:4625-33. doi:10.1210/en.2009-0119

19. Assini JM, Mulvihill EE, Burke AC, Sutherland BG, Telford DE, Chhoker SS, et al. Naringenin prevents obesity, hepatic steatosis, and glucose intolerance in male mice independent of fibroblast growth factor 21. Endocrinology (2015) 156:2087-102. doi:10.1210/en.2014-2003

20. Coskun T, Bina HA, Schneider MA, Dunbar JD, Hu CC, Chen Y, et al. Fibroblast growth factor 21 corrects obesity in mice. Endocrinology (2008) 149:6018-27. doi:10.1210/en.2008-0816

21. Xu J, Stanislaus S, Chinookoswong N, Lau YY, Hager T, Patel J, et al. Acute glucose-lowering and insulin-sensitizing action of FGF21 in insulin-resistant mouse models - association with liver and adipose tissue effects. Am J Physiol Endocrinol Metab (2009) 297:E1105-14. doi:10.1152/ajpendo.00348.2009

22. Adams AC, Coskun T, Rovira AR, Schneider MA, Raches DW, Micanovic R, et al. Fundamentals of FGF19 \& FGF21 action in vitro and in vivo. PLoS One (2012) 7:e38438. doi:10.1371/journal.pone.0038438

23. Kharitonenkov A, Shiyanova TL, Koester A, Ford AM, Micanovic R, Galbreath EJ, et al. FGF-21 as a novel metabolic regulator. J Clin Invest (2005) 115:1627-35. doi:10.1172/JCI23606

24. De Sousa-Coelho AL, Marrero PF, Haro D. Activating transcription factor 4-dependent induction of FGF21 during amino acid deprivation. Biochem J (2012) 443:165-71. doi:10.1042/BJ20111748

25. Pissios P, Hong S, Kennedy AR, Prasad D, Liu FF, Maratos-Flier E. Methionine and choline regulate the metabolic phenotype of a ketogenic diet. Mol Metab (2013) 2:306-13. doi:10.1016/j.molmet.2013.07.003

26. Laeger T, Henagan TM, Albarado DC, Redman LM, Bray GA, Noland RC, et al. FGF21 is an endocrine signal of protein restriction. J Clin Invest (2014) 124:3913-22. doi:10.1172/JCI74915
27. Koonen DP, Jacobs RL, Febbraio M, Young ME, Soltys CL, Ong H, et al. Increased hepatic CD36 expression contributes to dyslipidemia associated with diet-induced obesity. Diabetes (2007) 56:2863-71. doi:10.2337/ db07-0907

28. Conti R, Mannucci E, Pessotto P, Tassoni E, Carminati P, Giannessi F, et al. Selective reversible inhibition of liver carnitine palmitoyl-transferase 1 by teglicar reduces gluconeogenesis and improves glucose homeostasis. Diabetes (2011) 60:644-51. doi:10.2337/db10-0346

29. Berglund ED, Li CY, Bina HA, Lynes SE, Michael MD, Shanafelt AB, et al. Fibroblast growth factor 21 controls glycemia via regulation of hepatic glucose flux and insulin sensitivity. Endocrinology (2009) 150:4084-93. doi:10.1210/ en.2009-0221

30. Liang Q, Zhong L, Zhang J, Wang Y, Bornstein SR, Triggle CR, et al. FGF21 maintains glucose homeostasis by mediating the cross talk between liver and brain during prolonged fasting. Diabetes (2014) 63:4064-75. doi:10.2337/ db14-0541

31. Stemmer K, Zani F, Habegger KM, Neff C, Kotzbeck P, Bauer M, et al. FGF21 is not required for glucose homeostasis, ketosis or tumour suppression associated with ketogenic diets in mice. Diabetologia (2015) 58:2414-23. doi:10.1007/s00125-015-3668-7

Conflict of Interest Statement: All studies were conducted by Eli Lilly and Company. All authors were employees of Eli Lilly and Company.

Copyright (c) 2016 Antonellis, Hayes and Adams. This is an open-access article distributed under the terms of the Creative Commons Attribution License (CC BY). The use, distribution or reproduction in other forums is permitted, provided the original author(s) or licensor are credited and that the original publication in this journal is cited, in accordance with accepted academic practice. No use, distribution or reproduction is permitted which does not comply with these terms. 